\title{
An oracle based method to compute a coupled equilibrium in a model of international climate policy
}

\author{
Laurent Drouet • Alain Haurie • \\ Francesco Moresino • Jean-Philippe Vial • \\ Marc Vielle • Laurent Viguier
}

Published online: 28 February 2007

(C) Springer-Verlag 2007

\begin{abstract}
This paper proposes a computational game-theoretic model for the international negotiations that should take place at the end of the period covered by the Kyoto protocol. These negotiations could lead to a self-enforcing agreement on a burden sharing scheme given the necessary global emissions limit that will be imposed when the real extent of climate change is known. The model assumes a non-cooperative behavior of the parties except for the fact that they will be collectively committed to reach a target on total cumulative emissions by the year 2050. The concept of normalized equilibrium, introduced by J.B. Rosen for concave games with coupled constraints, is used to characterize
\end{abstract}

Partly supported by GICC (French Ministry of Ecology), TOCSIN (EU-044287) and the Swiss-NSF NCCR-Climate program of the Swiss NSF. For helpful comments and discussions, we thank A. Bernard, P. Thalmann, and the anonymous referee.

\section{Drouet $(\bowtie)$}

ORDECSYS and REME-EPFL, Ecole Polytechnique Fédérale de Lausanne,

Lausanne, Switzerland

e-mail: laurent.drouet@epfl.ch

A. Haurie · J.-P. Vial

ORDECSYS and University of Geneva, Geneva, Switzerland

M. Vielle

CEA-LERNA, University of Social Sciences, Toulouse, France

e-mail: mvielle@cict.fr

F. Moresino $\cdot$ M. Vielle $\cdot$ L. Viguier

REME-EPFL, Ecole Polytechnique Fédérale de Lausanne, Lausanne, Switzerland

e-mail: laurent.viguier@epfl.ch

L. Viguier

MIT Joint Program on the Science and Policy of Global Change, Cambridge, MA, USA 
a family of dynamic equilibrium solutions in an $m$-player game where the agents are (groups of) countries and the payoffs are the welfare gains obtained from a Computable General Equilibrium (CGE) model. The model deals with the uncertainty about climate sensitivity by computing an $S$-adapted equilibrium. These equilibria are computed using an oracle-based method permitting an implicit definition of the payoffs to the different players, obtained through simulations performed with the global CGE model GEMINI-E3.

Keywords Climate change negotiations - Dynamic game model Coupled constraints · Stochastic modal jumps

\section{Introduction}

In this paper we use a computational management science approach to study the strategic interactions of groups of countries when they shall negotiate the sharing of burden to stabilize the long term concentration of greenhouse gases (GHG). More precisely we develop a dynamic game model where the strategies of each player (state or group of countries) refer to the timing of emissions abatement and the payoffs are obtained in terms of welfare gains (or losses), after implementation of an international emissions trading scheme, compared with the business as usual (BAU) situation. These welfare gains are obtained through simulations performed with a Computable General Equilibrium (CGE) model. In this game a coupled constraint is imposed on all players together in order to limit the total emissions over the whole planning horizon. Games with coupled constraints have been first studied by Rosen (1965) who showed that a whole family (manifold) of equilibrium outcomes should be expected, indexed over a set of weights attributed to the players. The appropriateness of the normalized equilibrium concept to deal with environmental games seems to have been first recognized by Haurie (1995) and further explored by Haurie and Zaccour (1995) and Haurie and Krawczyk (1997). In this paper we explore the manifold of normalized equilibria both in a deterministic and in a stochastic framework about the scientific knowledge concerning the climate sensitivity. ${ }^{1}$

This game theoretic approach sheds a new light on the possible terms of the post Kyoto negotiations on climate change and the role that developing countries could have to play in long term climate change mitigation policy. The use of a game theoretic model instead of a global optimization one is justified by the following considerations: Provision of global public goods such as climate change mitigation must be voluntary since states or groups of countries can be induced but not forced to contribute to the global reduction of GHG emissions. In other words, international climate agreements must be self-enforcing. This condition has often been related to the characterization of

\footnotetext{
1 The climate sensitivity $C S$ parameter determines the surface atmospheric temperature change resulting from a doubling of GHG concentration compared with the preindustrial level.
} 
policies that constitute a Nash equilibrium in a non-cooperative game for the nations involved in the climate negotiation process. For example several authors have used game theoretic paradigms to study incentives based on issues linkages consisting in exchanging concessions across different policy dimensions (Carraro and Siniscalco 1996; Buchner et al. 2005). We explore the possibility to build self-enforcing agreements on the timing of emission caps for the different parties corresponding to different world regions. For that purpose we propose a dynamic game model which assumes a non-cooperative behavior of the parties except for the fact that they will be collectively committed to reach a target on total cumulative emissions by the year 2050 .

The dynamic game structure corresponds to the nature of the global climate challenge. As stated in the United Nations Framework Convention on Climate Change (UNFCCC), the ultimate goal is the "stabilization of greenhouse gas concentrations in the atmosphere at a level that would prevent dangerous anthropogenic human induced interference with the climate". Based on the assessment of climate impacts of global mean temperature rise, scientists confirm previous reports that, globally aggregated, the danger level begins once global mean temperature rises $2^{\circ} \mathrm{C}$ above preindustrial levels. In the deterministic version of the model one assumes that the sensitivity of the climate system to radiative forcing is known and implies that the $\mathrm{CO}_{2}$ concentration should be stabilized at no more than 550 parts per million by volume (ppmv). In the stochastic version of the model one assumes that there is still a global uncertainty on the real value of the $C S$ parameter. Depending on this value different global limits on cumulative emissions will be imposed on all countries together in order that the concentration levels remain within a tolerable window in terms of environmental and ecological damage. ${ }^{2}$ Then the different parties in an agreement behave as non-cooperative players, even though they realize that a global cumulative emissions constraint is binding all countries together in the long run.

Achieving this target will be a major challenge that will require a participation of large developing countries since they constitute an important potential source of GHG emissions in the future. As said in a recent EU communique,

An effective future multilateral climate change regime will require all major emitters to contribute by limiting or reducing their greenhouse gas emissions. Without the participation of other developed countries and key emitters among developing countries, substantial cuts in EU emissions alone will fail to achieve the $2^{\circ} \mathrm{C}$ target.

States and regions have thus to agree on the long term GHG concentration target to reach collectively. Given this common target each (group of) nation's respective contribution to the international effort will be guided by its own interest, if the agreement has to be self-enforcing. The battle against global warming is thus a mixture of cooperative (attainment of a common goal) and non-cooperative (economic selfishness) behavior. In that context, international

2 See Toth (2005) for a discussion of the tolerable window concept. 
GHG emissions trading might be used as a mean to reduce the economic costs of the emission constraint, and to create incentives for participation through financial transfers. Once the global goal is defined the degrees of freedom left to the players reside in the timing of their abatement commitments. The normalized equilibrium concept finds a solution associated with a weighting of the different players. For example if one increases the weight given to DCs, the share of burden for these nations in satisfying the global constraint is reduced and the sequence of abatement is obtained as a best reply to the decisions made by other (groups of) countries. Therefore, in addition to the global aim dictated by the climate dynamics, the negotiation will also have to decide the weighting given to the different groups of nations that will be parties in the agreement. In this paper we compute the manifold of normalized equilibria when we consider only 3 groups of nations, USA, rest of OECD (also called IND), DCs (also called SUD), and when one varies the weighting given to these players in the equilibrium definition. The model used is a two-level dynamic game where governments try to get the best agreement for their respective economy knowing that, once quotas are fixed, the economic agents will be confronted to market competition for $\mathrm{GHG}$ emission quotas.

From an Operations Research perspective, the paper implements an oracle based technique to solve variational inequalities. The oracle here is the CGE that computes the welfare gains to the different players and their sensitivities to emission cap decisions. The technique converts the variational inequalities into a kind of convex feasibility problem, which can be solved, with known complexity, via the homogeneous analytic center cutting plane method (ACCPM) (Nesterov and Vial 1999). In the present paper, we use the variant described in Babonneau et al. (2006) of the original non-homogenous version of ACCPM (Goffin et al. 1992). Its behavior is quite similar to the homogenous one, but its complexity for variational inequalities is not established. In our experiments, the non-homogenous version performs very well.

It is important to notice that the game we solve is about allocations rather than a game on the overall target. We assume that a $2^{\circ}$ target would be accepted by all countries of the world (although one may expect that a common agreed global target will probably be the crux of the matter during the second commitment period negotiations). We model a coupled constraint on total emissions and let the players play noncooperatively on the timing and allocation of their respective shares of this overall burden. We do not claim that we define levels of emission reduction that leave every player better off when playing the game than staying out of the game.

The paper is organized as follows: the structure of the coupled game model is presented in Sect. 2; in Sect. 3, we briefly present the multi-sector and multicountry CGE model of the world economy that is used for numerical simulations; the Analytic Center Cutting Plane Method (ACCPM) implemented to solve the weak variational inequality problem is described in Sect. 4; in Sect. 5 we give the numerical results obtained for a case study where countries have to decide on their own abatement level under a global target on cumulative GHG emissions by 2050 which is consistent with a commitment to limit global 
temperature rise to $2^{\circ} \mathrm{C}$ above pre-industrial levels; in Sect. 6, we conclude and envision future extensions of this model.

\section{The model}

In this section we present a stochastic game model reduced to its simplest formulation with only two periods, the first one corresponding to the commitments to be negotiated in the period 2010-2025, and the second one corresponding to the recourse to be played by the actors in the $2025-2050$ period when the real severity of climate change will be known.

\subsection{Players, moves and payoffs}

The game is played over 2 periods $t=0,1$. $M$ is a set of $m$ groups of countries hereafter called players which must decide on the caps they impose on their respective global emissions of GHGs in each period. Let $\Omega$ be the set of possible realizations of the climate sensitivity (CS) parameter values. We represent the uncertainty on this value as an event tree as shown in Fig. 1.

Let $\pi(\omega)$ be the probability of realization $\omega \in \Omega$. We denote $\bar{e}_{j}(t, \omega)$ the cap decided by player $j$ for period $t$ and CS $\omega \in \Omega$. In period 0 the CS is unknown, therefore the following equalities must be satisfied:

$$
\bar{e}_{j}(0, \omega)=\bar{e}_{j}\left(0, \omega^{\prime}\right) \quad \forall j \in M, \forall \omega, \omega^{\prime} \in \Omega \text {. }
$$

In period 1 the CS is known. and each player adapts its decision $\bar{e}_{j}(1, \omega)$ to the observed realization $\omega$. Depending on the realization $\omega \in \Omega$, a global limit $\bar{E}(\omega)$ will be imposed on the cumulative emissions of both periods $t=0,1$. Therefore the following coupled constraints are binding all players together:

$$
\sum_{j \in M} \sum_{t=0}^{1} \bar{e}_{j}(t, \omega) \leq \bar{E}(\omega) \quad \forall \omega \in \Omega
$$

Fig. 1 Climate sensitivity uncertainty

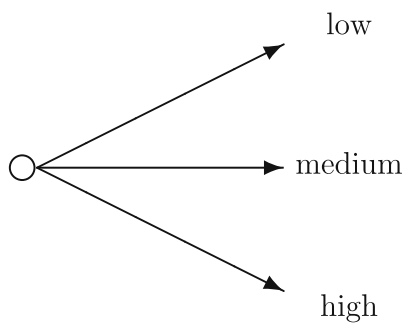


Let $\overline{\mathbf{e}}(t, \omega)=\left\{\bar{e}_{j}(t, \omega)\right\}_{j \in M}$ denote the vector of emissions caps for all players in period $t$, and CS value $\omega$. Given these cap values a general economic equilibrium is computed for the $m$-country which determines a welfare gain for each player, hereafter called its payoff at $t, \omega$ and denoted $W_{j}(\overline{\mathbf{e}}(t, \omega))$. Given a choice of emission programs $\overline{\mathbf{e}}=\{\overline{\mathbf{e}}(t, \omega) \mid: t=0,1 ; \omega \in \Omega\}$ the expected payoff to player $j$ is given by

$$
J_{j}(\overline{\mathbf{e}})=\sum_{t=0}^{1} \sum_{\omega \in \Omega} \pi(\omega) W_{j}(\overline{\mathbf{e}}(t, \omega)) \quad j \in M .
$$

\subsection{Normalized equilibrium solutions}

We assume that the players behave in a noncooperative way but are bound to satisfy the global cumulative emissions constraints (2) that are contingent to the realization $\omega$ of the CS. The solution concept that we propose to use is an adaptation of the so-called $S$-adapted equilibrium solution introduced in Haurie et al. (1990) and further studied in Haurie and Viguier (2003) and Haurie and Moresino (2002). It is also related to the concept of normalized equilibrium introduced by Rosen (1965) to deal with games where the players are bound by a coupled constraint. In the $S$-adapted information structure one assumes that the players adapt their decisions to the revelation of the uncertain parameter value. However one does not assume that the players can adapt their decisions to the history of the game, i.e. to the decisions taken by the other players in previous periods.

Let us call $\mathcal{E}$ the set of emissions $\overline{\mathbf{e}}$ that satisfy the constraints(1) and (2). Denote also $\left[\overline{\mathbf{e}}^{*-j}, \bar{e}_{j}\right]$ the emission program obtained from $\overline{\mathbf{e}}^{*}$ by replacing only the emission programm $\bar{e}_{j}^{*}$ of player $j$ by $\bar{e}_{j}$.

Definition 1 The emission program $\overline{\mathbf{e}}^{*}$ is an equilibrium under the coupled constraints (2) if the following holds for each player $j \in M$ :

$$
\begin{aligned}
\overline{\mathbf{e}}^{*} & \in \mathcal{E} \\
J_{j}\left(\overline{\mathbf{e}}^{*}\right) & \geq J_{j}\left(\left[\overline{\mathbf{e}}^{*-j}, \bar{e}_{j}\right]\right) \quad \forall \bar{e}_{j} \text { s.t. }\left[\overline{\mathbf{e}}^{*-j}, \bar{e}_{j}\right] \in \mathcal{E} .
\end{aligned}
$$

Therefore, in this equilibrium, each player replies optimally to the emission program chosen by the other players, under the constraint that the global cumulative emission limits must be respected.

It is possible to characterize a class of such equilibria through a fixed point condition for a best reply mapping defined as follows. Let $\mathbf{r}=\left(r_{j}\right)_{j \in M}$ with $r_{j}>0$ and $\sum_{j \in M} r_{j}=1$ be a given weighting of the different players. Then introduce the combined response function

$$
\theta\left(\overline{\mathbf{e}}^{*}, \overline{\mathbf{e}} ; \mathbf{r}\right)=\sum_{j \in M} r_{j} J_{j}\left(\left[\overline{\mathbf{e}}^{*-j}, \bar{e}_{j}\right]\right)
$$


It is easy to verify that, if $\overline{\mathbf{e}}^{*}$ satisfies the fixed point condition

$$
\theta\left(\overline{\mathbf{e}}^{*}, \overline{\mathbf{e}}^{*} ; \mathbf{r}\right)=\max _{\overline{\mathbf{e}} \in \mathcal{E}} \theta\left(\overline{\mathbf{e}}^{*}, \overline{\mathbf{e}} ; \mathbf{r}\right)
$$

then it is an equilibrium under the coupled constraint.

Definition 2 The emission program $\overline{\mathbf{e}}^{*}$ is a normalized equilibrium if it satisfies (7) for a weighting $\mathbf{r}$ and a combined response function defined as in (6).

The RHS of (7) defines an optimization problem under constraint. Assuming the required regularity we can introduce a Kuhn-Tucker multiplier $\lambda^{o}(\omega)$ for each constraint $\sum_{t=0}^{1} \bar{e}_{j}(t, \omega) \leq \bar{E}(\omega)$ and form the Lagrangian

$$
L=\theta\left(\overline{\mathbf{e}}^{*}, \overline{\mathbf{e}} ; \mathbf{r}\right)+\sum_{\omega \in \Omega} \lambda^{o}(\omega)\left(\bar{E}(\omega)-\sum_{j \in M} \sum_{t=0}^{1} \bar{e}_{j}(t, \omega)\right)
$$

Therefore, by applying the standard K-T optimality conditions we can see that the normalized equilibrium is also the Nash equilibrium solution for an auxiliary game with a payoff function defined for each player $j$ by

$$
J_{j}(\overline{\mathbf{e}})+\sum_{\omega \in \Omega} \lambda^{j}(\omega)\left(\bar{E}(\omega)-\sum_{j \in M} \sum_{t=0}^{1} \bar{e}_{j}(t, \omega)\right),
$$

where

$$
\lambda^{j}(\omega)=\frac{1}{r_{j}} \lambda^{o}(\omega), \quad \omega \in \Omega .
$$

This characterization has an interesting interpretation in terms of negotiation for a climate change policy. A common "tax" $\lambda^{o}(\omega)$ is defined and applied to each player with an intensity $\frac{1}{r_{j}}$ that depends on the weight given to this player in the global response function.

\section{Getting the payoffs via GEMINI-E3}

\subsection{General overview}

The payoffs of the game are computed using GEMINI-E3 which is a dynamic-recursive CGE describing the world economy in 21 regions with 14 sectors, and which incorporates a highly detailed representation of indirect taxation (Bernard and Vielle 1998). This version of GEMINI-E3 is formulated as a Mixed Complementarity Problem (MCP) using GAMS with the PATH solver (Ferris and Munson 2000; Ferris and Pang 1997). GEMINI-E3 is built on a comprehensive energy-economy data set, the GTAP-5 database (Hertel 1997), that expresses a consistent representation of energy markets in physical units as 
well as a detailed Social Accounting Matrix (SAM) for a large set of countries or regions and bilateral trade flows. It is the fourth GEMINI-E3 version that has been especially designed to calculate the social marginal abatement costs (MAC), i.e. the welfare loss of a unit increase in pollution abatement (Bernard and Vielle 2003). The original version of GEMINI-E3 is fully described in Bernard and Vielle (1998). ${ }^{3}$ Updated versions of the model have been used to analyze the implementation of economic instruments for GHG emissions in a second-best setting (Bernard and Vielle 2000), to assess the strategic allocation of GHG emission allowances in the EU-wide market (Bernard et al. 2005) and to analyze the behavior of Russia in the Kyoto Protocol (Bernard et al. 2003, 2005).

For each sector the model computes the demand on the basis of household consumption, government consumption, exports, investment, and intermediate uses. Total demand is then divided between domestic production and imports, using the Armington assumption (Armington 1969). Under this convention a domestically produced good is treated as a different commodity from an imported good produced in the same industry. Production technologies are described using nested CES functions.

\subsection{Welfare cost}

Household's behavior consists in three interdependent decisions: (1) labor supply; (2) savings; and (3) consumption of the different goods and services. In GEMINI-E3, we suppose that labor supply and the rate of saving are exogenously fixed. The utility function corresponds to a Stone-Geary utility function (Stone 1983) which is written as

$$
u_{r}=\sum_{i} \beta_{i r} \cdot \ln \left(\mathrm{HC}_{i r}-\phi_{i r}\right)
$$

where $\mathrm{HC}_{i r}$ is the household consumption of product $i$ in region $r, \phi_{i r}$ represents the minimum necessary purchases of good $i$, and $\beta_{i r}$ corresponds to the marginal budget share of good $i$. Maximization under budgetary constraint

$$
\mathrm{HCT}_{r}=\sum_{i} \mathrm{PC}_{i r} \cdot \mathrm{HC}_{i r}
$$

yields

$$
\mathrm{HC}_{i r}=\phi_{i r}+\frac{\beta_{i r}}{\mathrm{PC}_{i r}} \cdot\left[\mathrm{HCT}_{r}-\sum_{k}\left(\mathrm{PC}_{k r} \cdot \phi_{k r}\right)\right],
$$

where $\mathrm{PC}_{i r}$ is the price of household consumption for product $i$ in region $r$.

\footnotetext{
3 For a complete description of the model see our web site and the technical document downloadable at: http://gemini-e3.epfl.ch/. 
The welfare cost of climate policies is measured comprehensively by changes in households' welfare since final demand of other institutional sectors is supposed unchanged in scenarios. Measurement of this welfare change is represented by the sum of the change in income and the "Compensative Variation of Income" $(C V I)$ of the change in prices, according to the classical formula. In the case of a Stone-Geary utility function, the $C V I$ for a change from an initial situation defined by the price system $\left(\overline{\mathrm{PC}}_{i r}\right)$ to a final situation $\left(\mathrm{PC}_{i r}\right)$ is such as

$$
\frac{\overline{\mathrm{HCT}}_{r}-\sum_{i} \overline{\mathrm{PC}}_{i r} \cdot \phi_{i r}}{\Pi_{i}\left(\overline{\mathrm{PC}}_{i r}\right)^{\beta_{i r}}}=\frac{\overline{\mathrm{HCT}}_{r}+C V I_{r}-\sum_{i} \mathrm{PC}_{i r} \cdot \phi_{i r}}{\Pi_{i}\left(\mathrm{PC}_{i r}\right)^{\beta_{i r}}} .
$$

The households' surplus is then given by

$$
S_{r}=\left(\mathrm{HCT}_{r}-\sum_{i} \mathrm{PC}_{i r} \cdot \phi_{i r}\right)-\Pi_{i}\left(\frac{\mathrm{PC}_{i r}}{\overline{\mathrm{PC}}_{i r}}\right)^{\beta_{i r}}\left(\overline{\mathrm{HCT}}_{r}-\sum_{i} \overline{\mathrm{PC}}_{i r} \cdot \phi_{i r}\right) .
$$

In summary, the CGE model associates a welfare gain (cost) for each country and each period, with a given emissions program $\overline{\mathbf{e}}$ which defines caps for all countries at each period. It is important to notice that these welfare gains are obtained under the assumption that an international emissions trading system is put in place. It should be noted that we do not take into account the welfare gain coming from the limitation of the global warming (i.e. the decrease of damage resulting of the climate change).

\section{Oracle-based optimization framework}

\subsection{Normalized equilibrium and variational inequality}

For concave games with differentiable payoff functions $J_{j}(\cdot), \overline{\mathbf{e}}^{*}$ is a normalized equilibrium if and only if it is a solution of the following variational inequality problem:

$$
\left\langle F\left(\overline{\mathbf{e}}^{*}\right), \overline{\mathbf{e}}^{*}-\overline{\mathbf{e}}\right\rangle \geq 0 \quad \forall \overline{\mathbf{e}} \in \mathcal{E},
$$

where $\langle\cdot, \cdot\rangle$ denotes the scalar product and the pseudogradient $F(\cdot)$ is defined by

$$
F(\overline{\mathbf{e}})=\left(\begin{array}{c}
r_{1} \nabla_{\bar{e}_{1}} J_{1}(\overline{\mathbf{e}}) \\
\vdots \\
r_{j} \nabla_{\bar{e}_{j}} J_{j}(\overline{\mathbf{e}}) \\
\vdots \\
r_{m} \nabla_{\bar{e}_{m}} J_{m}(\overline{\mathbf{e}})
\end{array}\right) .
$$

It has been proved in Rosen (1965) that a normalized equilibrium exists if the payoff functions $J_{j}(\cdot)$ are continuous in $\overline{\mathbf{e}}$ and concave in $\bar{e}_{j}$ and if $\mathcal{E}$ is compact. In the same reference it is proved also that the normalized equilibrium is unique 
if the function $-F(\cdot)$ is strictly monotone, i.e. if the following holds:

$$
\left\langle F\left(\overline{\mathbf{e}}^{2}\right)-F\left(\overline{\mathbf{e}}^{1}\right), \overline{\mathbf{e}}^{1}-\overline{\mathbf{e}}^{2}\right\rangle>0 \quad \forall \overline{\mathbf{e}}^{1}, \overline{\mathbf{e}}^{2} \in \mathcal{E} .
$$

Solving (16) is a most challenging problem. When $-F$ is monotone and continuous or when it is maximal monotone, the strong form of the variational inequality (16) is known to be equivalent to the so-called weak form (see Chap. 7 in Nesterov and Nemirovskii 1994)

$$
\left\langle F(\overline{\mathbf{e}}), \overline{\mathbf{e}}^{*}-\overline{\mathbf{e}}\right\rangle \geq 0 \quad \forall \overline{\mathbf{e}} \in \mathcal{E} .
$$

One can associate to the weak form, the so-called dual-gap function

$$
\phi(\overline{\mathbf{e}})=\min _{\overline{\mathbf{e}}^{\prime} \in \mathcal{E}}\left\langle F\left(\overline{\mathbf{e}}^{\prime}\right), \overline{\mathbf{e}}-\overline{\mathbf{e}}^{\prime}\right\rangle .
$$

This function is concave and non-positive. It achieves a maximum value 0 at any solution point of (19). Finding a solution to the weak variational inequality problem thus amounts to maximizing a concave function. Before presenting a solution method, we point out that we cannot expect from such a method to find an exact solution. Therefore, we must be satisfied with an approximate solution. We say that $\overline{\mathbf{e}}$ is an $\epsilon$-approximate weak solution if

$$
\phi(\overline{\mathbf{e}}) \geq-\epsilon .
$$

\subsection{An oracle method to solve the variational inequality problem}

The trouble with the gap function is that it is not computable. Indeed, solving the minimization problem in (20) is a nonconvex programming problem that is likely to be as difficult as the original strong variational inequality problem (16). To circumvent the difficulty, we consider that (21) defines the convex feasibility problem

$$
\text { Find } \overline{\mathbf{e}} \in \mathcal{E}_{\epsilon}^{*}=\{\overline{\mathbf{e}} \in Q \mid \phi(\overline{\mathbf{e}}) \geq-\epsilon\} \text {. }
$$

Since $\phi$ is concave, $\mathcal{E}_{\epsilon}^{*}$ is convex. If $\epsilon=0$, the set $\mathcal{E}^{*}=\mathcal{E}_{0}^{*}$ is the set of solutions to the weak variational inequality.

We can associate to $\mathcal{E}^{*}$ an oracle that provides at any $\overline{\mathbf{e}}$ the separation hyperplane

$$
\left\langle F(\overline{\mathbf{e}}), \overline{\mathbf{e}}^{*}-\overline{\mathbf{e}}\right\rangle \geq 0 \quad \forall \overline{\mathbf{e}}^{*} \in \mathcal{E}^{*}
$$

We shall use this property to implement the Analytic Center Cutting Plan Method (ACCPM) (Goffin et al. 1992) to solve the feasibility problem (22). This algorithm proceeds as follows. Let $\left\{\overline{\mathbf{e}}^{k}\right\}_{k \leq K}$ be a series of so-called query points. We associate to this series the localization set

$$
\mathcal{L}_{K}=\left\{\overline{\mathbf{e}} \in \mathcal{E} \mid\left\langle F\left(\overline{\mathbf{e}}^{k}\right), \overline{\mathbf{e}}-\overline{\mathbf{e}}^{k}\right\rangle \geq 0, k=1, \ldots, K\right\}
$$


Note that $\mathcal{E}^{*} \subset \mathcal{L}_{K}$. Recall that in our problem of interest $\mathcal{E}$ is a bounded polyhedral set. For convenience, we give it the compact form $\mathcal{E}=\{\overline{\mathbf{e}} \mid A \overline{\mathbf{e}} \leq b\}$. In ACCPM, the next query point is selected so as to minimize the logarithmic barrier function

$$
B_{\mathcal{L}_{K}}(\overline{\mathbf{e}})=-\sum_{j} \log (b-A \overline{\mathbf{e}})_{j}-\sum_{k \leq K} \log \left(\left\langle F\left(\overline{\mathbf{e}}^{k}\right), \overline{\mathbf{e}}-\overline{\mathbf{e}}^{k}\right\rangle\right) .
$$

If $\mathcal{L}_{K}$ has a non-empty interior, the barrier function is well-defined and achieves its minimum at a unique point, named the analytic center.

Prior to stating the algorithm, we point out two difficulties. The first one is linked to the nature of the variational inequality problem: we lack a computable termination criterion because the dual gap function $\phi(\overline{\mathbf{e}})$ is not computable. We propose instead to use the computable condition

$$
\left\langle F\left(\overline{\mathbf{e}}^{K}\right), \overline{\mathbf{e}}-\overline{\mathbf{e}}^{K}\right\rangle \geq-\epsilon \quad \forall \overline{\mathbf{e}} \in \mathcal{E} .
$$

Note that this criterion can be checked by solving a simple linear programming problem. If the criterion is met, it certifies that the point is an approximate solution to the strong variational inequality. The other difficulty is related to the computation of $F$ in our game problem of interest. In the game that has been defined in the previous section, the payoffs are not defined analytically but are revealed by simulations performed via a CGE model. Therefore we had to implement an oracle-based method where the CGE model, hereafter called the "oracle", is queried at each iteration. The oracle returns an information consisting of (a) the evaluation of the payoff values and, (b) the evaluation of the pseudogradient at the query point. In the implementation, we evaluate the pseudogradient at each query point using finite differences.

We can now state the ACCPM algorithm for the game problem

Step 0 (initialization) Set $k=0$ and the localization set $\mathcal{L}_{0}=\mathcal{E}$. Choose $\epsilon>0$.

Step 1 (computation of the analytical center)

Compute $\overline{\mathbf{e}}^{k}=\arg \min _{\overline{\mathbf{e}}} B_{\mathcal{L}_{K}}(\overline{\mathbf{e}})$ the analytical center of the localization set $\mathcal{L}_{k}$.

Step 2 (computation of the pseudogradient)

Compute $F\left(\overline{\mathbf{e}}^{k}\right)$.

Step 3 (stopping criterion)

Solve the linear programming problem $t=\min \left\{\left\langle F\left(\overline{\mathbf{e}}^{k}\right), \overline{\mathbf{e}}-\overline{\mathbf{e}}^{k}\right\rangle \mid \overline{\mathbf{e}} \in \mathcal{E}\right\}$.

IF $t \geq-\epsilon$ STOP.

Step 4 (generation of a cutting plane)

Update $\mathcal{L}_{k+1}=\mathcal{L}_{k} \cap\left\{\overline{\mathbf{e}} \mid\left\langle F\left(\overline{\mathbf{e}}^{k}\right), \overline{\mathbf{e}}-\overline{\mathbf{e}}^{k}\right\rangle \geq 0\right\}$.

Increment $k$ and GO TO STEP 1 .

It has been shown (Luo et al. 1996) that ACCPM solves the convex feasibility problem in pseudo-polynomial time. Notwithstanding the fact that we can only approximate $F$, some basic assumptions of Luo et al. (1996) are not satisfied. Even if we knew the exact value of $F$ and assumed that $F$ is continuous and 
monotone, our oracle does not separate the solution set $\phi(\overline{\mathbf{e}}) \geq-\epsilon$ from the current query point (we can only separate the point from $\mathcal{E}^{*}$ ). However, the good point is that when our algorithm stops with the test $t \geq-\epsilon$, we are sure that the current point is an $\epsilon$-approximate solution to the strong variational inequality problem.

We conclude this section by mentioning that there exists a homogeneous version of ACCPM (Nesterov and Vial 1999) with a polynomial complexity estimate to compute a weak solution under the assumption that $F$ is monotone. We have not implemented this version.

\section{Numerical results}

\subsection{Deterministic case}

As shown in Fig. 2, global GHG emissions are expected to be close to 15 Giga tons of Carbon equivalent ( $\mathrm{GtC}$-eq) in the business-as-usual scenario.

Our policy scenarios are built on the objective of keeping global mean surface warming relative to preindustrial below $2^{\circ}$. The two-degree target has recently been put forward by some nations, scientists and environmental organizations. For example, the European Union endorsed the objective of limiting mean global warming to below $2^{\circ} \mathrm{C}$ over pre-industrial levels to avoid serious climate change impacts. This translates into stabilization of carbon concentration toward $550 \mathrm{ppmv}$ and requires to limit global emissions to about $11 \mathrm{GtC}$-eq in 2050. It represents a $-30 \%$ reduction target, and around $480 \mathrm{Gt}$ of GHG emission quotas to share in the whole period of time. Figure 2 also presents the global GHG emission trajectory under the unique coupled equilibrium obtained when the same weight is put on the three players.

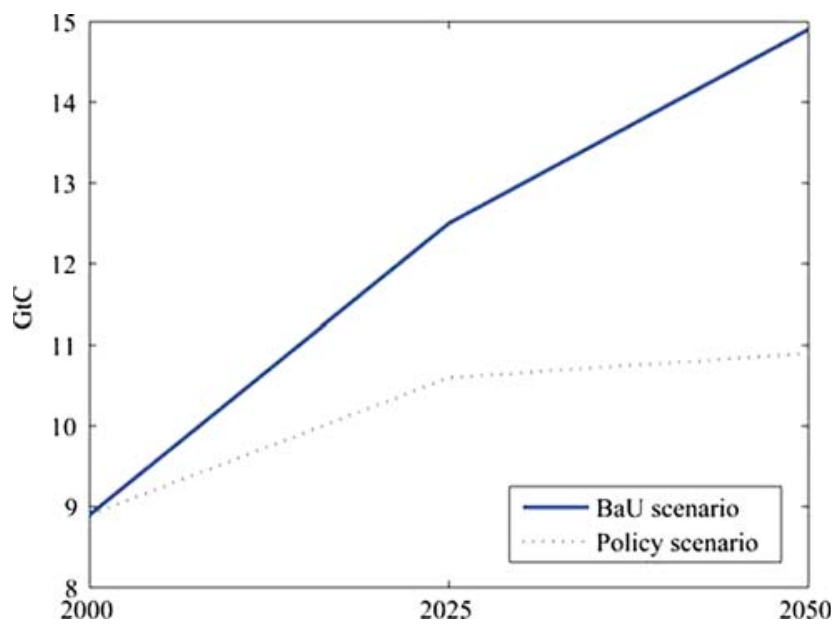

Fig. 2 Baseline and policy-constrained global GHG emissions, 2000-2050 (in GtC-eq) 


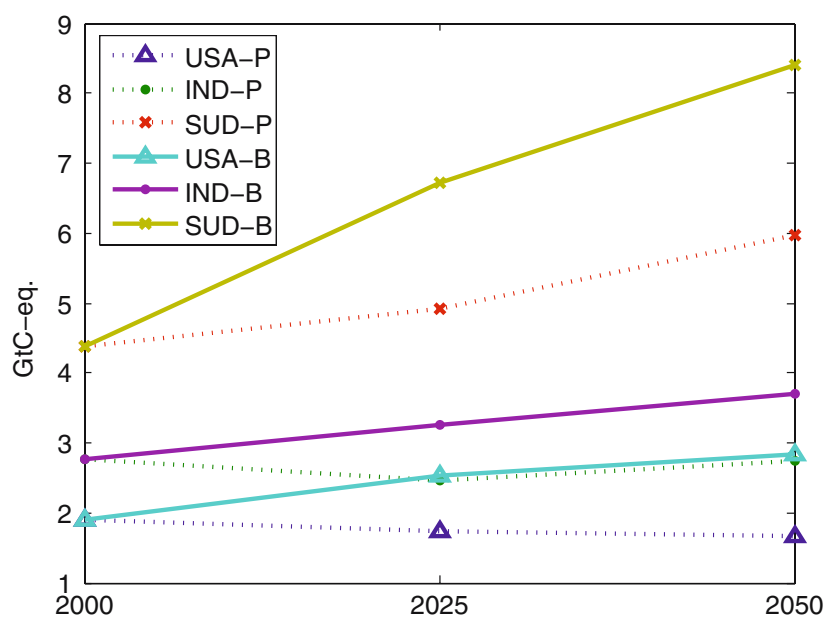

Fig. 3 GHG emission quotas at the coupled equilibrium, 2000-2050 (in GtC-eq)

Figure 3 depicts the burden sharing obtained when the same weight is put on the three players. Considering the global constraint on GHG emissions by 2050 , the USA, the other industrialized economies, and the developing world would be committed to reduce their GHG emissions by $-33,-26$, and $-47 \%$, respectively. As shown on the graph, the players do not delay abatement at the equilibrium but decide to progressively reduce their emissions. Given these unilateral decisions on abatement, the GHG price increase smoothly from 108 $\$ / \mathrm{tC}$-eq in 2025 to $160 \$ / \mathrm{tC}$-eq in 2025.

Figure 4 gives the payoffs of the players at the equilibrium. The payoffs are the total discounted welfare costs associated with the GHG emission constraint in each region for the whole period of time. In absolute terms, total costs are

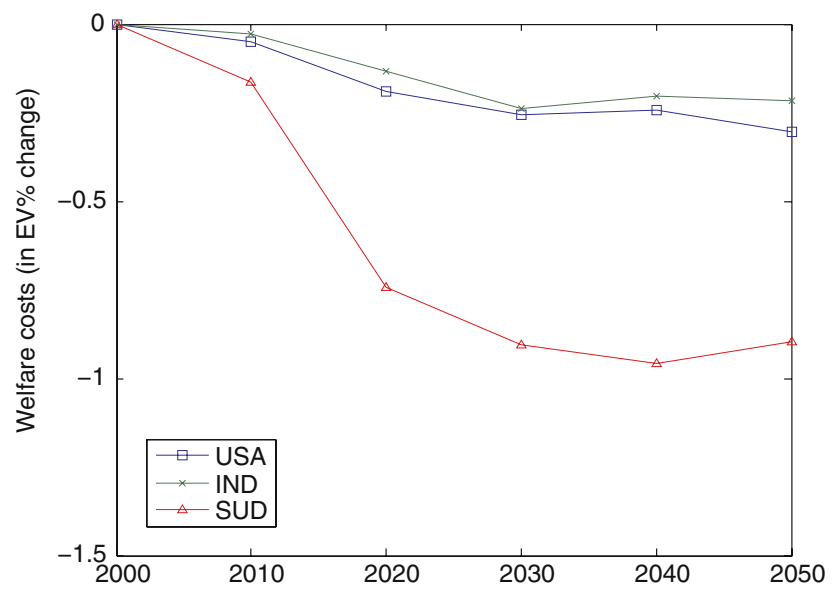

Fig. 4 Welfare costs by region, 2000-2050 (in EV\% change) 
almost the same for USA and IND; but the non-cooperative equilibrium is costly for DCs which support $60 \%$ of the global welfare cost. In the year 2050 , the equivalent variation of income (see definition in section 3 ) due to to the climate policy is around $-0.3,-0.2$, and $-0.9 \%$ for the US, the other OECD countries and developing countries, respectively.

\subsection{Sensitivity analysis}

As explained in Rosen (1965), the possibly unique normalized equilibrium should change with the weighting $\mathbf{r}$ of the different players. In the previous section the same weight was put on the three players of the game. It is completely arbitrary, and probably a too strong assumption. To reflect the negotiating power of the players, the weighting of the players might be defined in consistency with distributive justice precludes or simply to reflect the negotiating power of the players. It could be based on population, macro-economic indicators (i.e. GDP, consumption) and/or environmental indicators (i.e. GHG emissions). It is not in the aim of this section to try to define the "right" weighting but to explore how equilibria might change with different weightings.

One simple test is to increase the weight of developing countries compared to the "balanced" policy case assessed in the previous section. As expected, this has the effect of reducing the cost of the climate policy for DCs (Fig. 5). One can define a weighting that would lead to an equilibrium where DCs do not support any welfare cost. This equilibrium does not correspond to the burden sharing option, advocated by IEA (2002), Philibert (2000), Philibert and Pershing (2001), where the baseline emissions are given to the DCs (also called the "non-constraining target" option). It is a situation where the costs

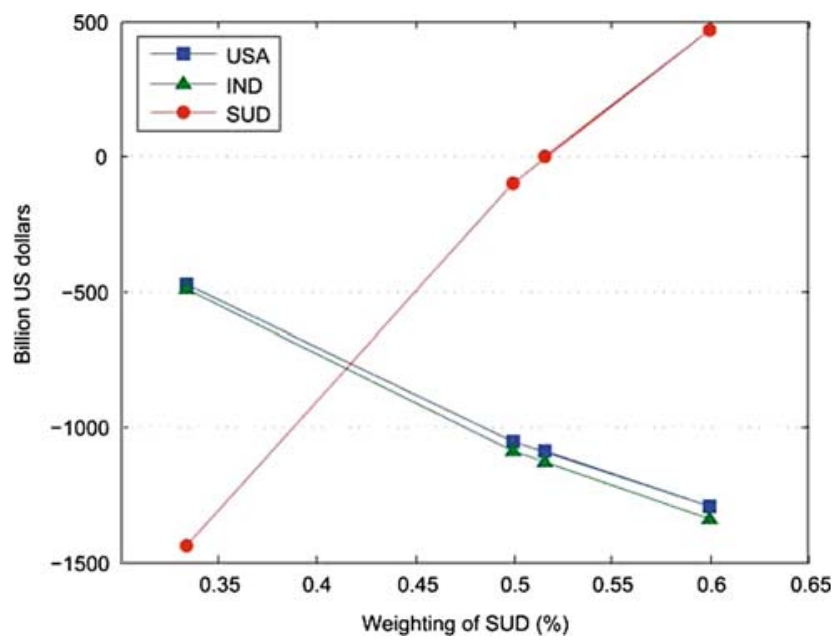

Fig. 5 North-south utility frontier based on discounted welfare costs for USA+IND and SUD, 2000-2050 (in billion USD) 


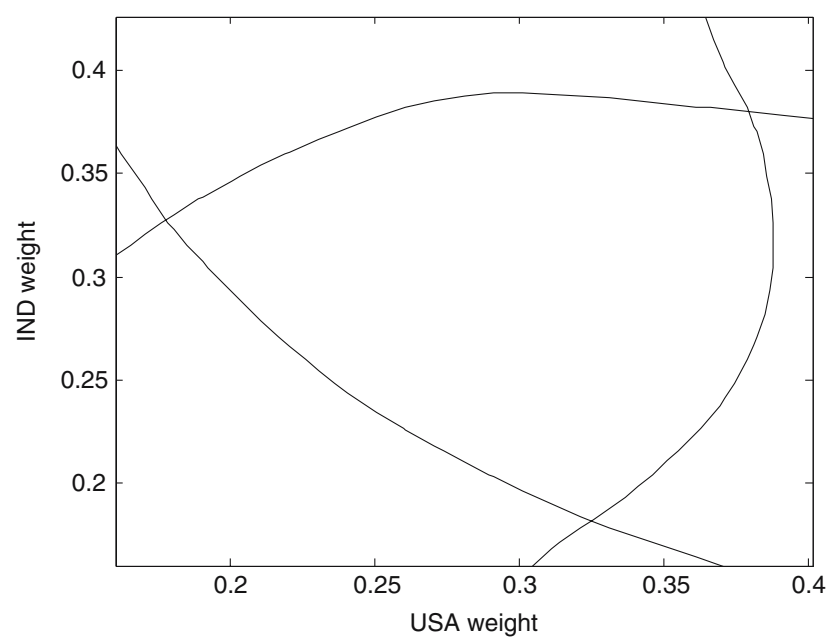

Fig. 6 Set of coupled equilibria

of meeting the domestic reduction targets are balanced by the gains associated with the selling of emission permits. Based on GEMINI-E3 estimates, we find that DCs might preserve their welfare growth while accepting a $20 \%$ reduction target (compared to their baseline). As shown in Fig. 5, DCs are better off when their weight increases above $53 \%$. They might voluntarily accept to reduce their GHG emissions in order to participate in international emission trading.

In Fig. 6 we explore the set of coupled equilibria that can be obtained from changing the weights of the different players. The $x$-axis represents the weight of the USA whereas the $y$-axis is for the weight of the other industrialized countries. The weight of DCs does not appear in this graph but the weights of the three players always sum to one. We assume that the players are not allowed to gain from the fight against global climate change. The weights can be changed to create incentive for participation and to define an acceptable allocation of the climate burden but the players are not supposed to be better off with the GHG emissions constraint than without. As depicted in Fig 5, the weights might range from 16 to $38 \%$ and 18 to $37 \%$ for the United States and the other industrialized countries, respectively. The weight of developing countries might go from 24 to $53 \%$.

The resulting manifold of South (SUD) equilibrium outcomes is shown in Fig. 7.

\subsection{Policy scenarios}

We use the smooth stabilization profiles (SP) constructed by Knutti et al. (2006). As shown in Fig. 8, the set of scenarios includes direct stabilization at levels of 450, 550, and 650 ppm atmospheric $\mathrm{CO}_{2}$ equivalent (SP450, SP550 and SP650). 


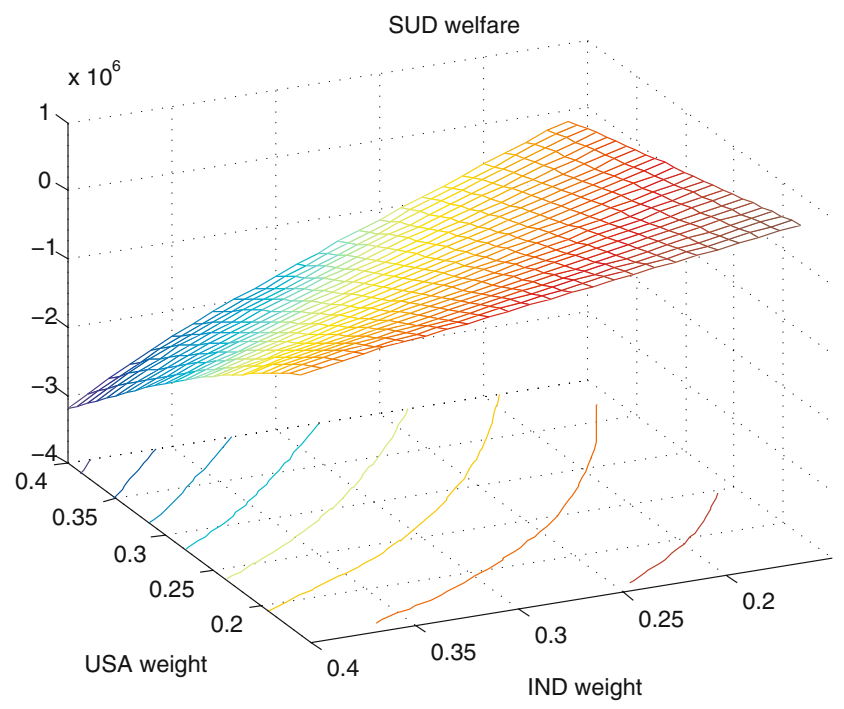

Fig. 7 South (SUD) manifold of equilibrium outcomes, 2000-2050 (in billion USD)

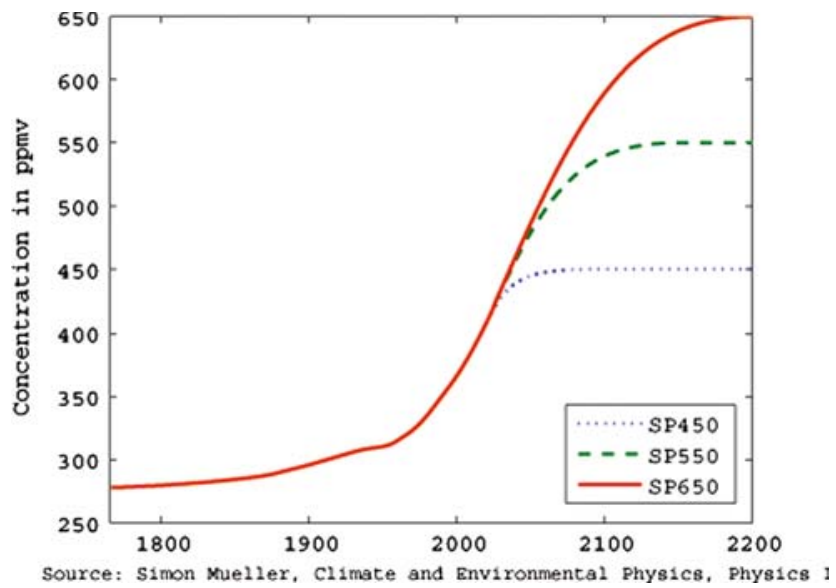

Fig. 8 Smooth stabilization profiles

The emission commitment scenarios depicted in Fig. 9 are calculated based on SP scenarios and the Bern carbon cycle model. ${ }^{4}$

In Knutti et al. (2006) an ensemble of probabilistic projections of future climate change have been performed for a range of $\mathrm{CO}_{2}$ stabilization profiles intended for the Fourth Assessment Report of the Intergovernmental Panel on Climate Change are presented. A very large ensemble of simulations with the reduced complexity, Bern 2.5D climate model was used to explore the uncertainties in projected long term changes in surface temperature and sea level due

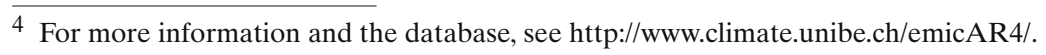




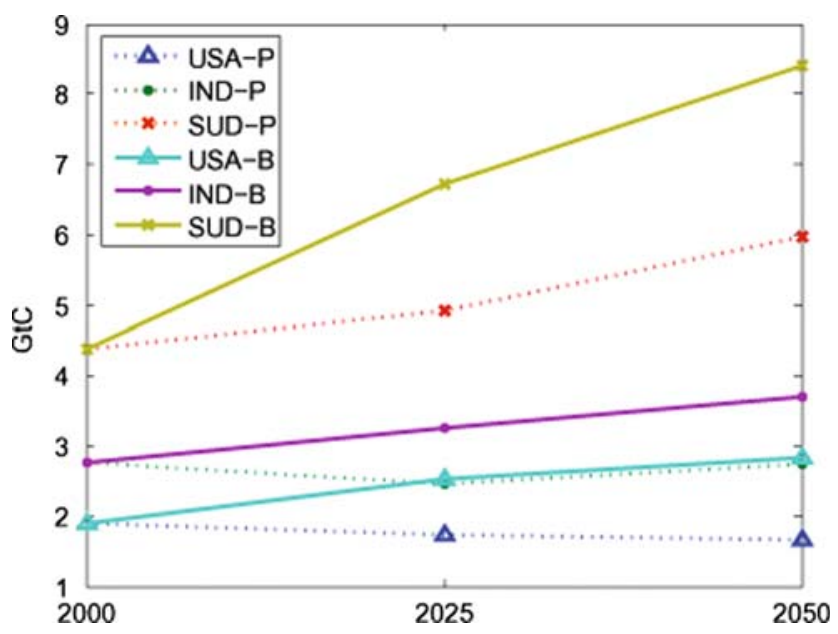

Fig. 9 Global emission profiles (in GtC)

Table 1 Probabilities of staying below $2{ }^{\circ} \mathrm{C}$ and global emission quotas with SP450, SP550, and SP650

\begin{tabular}{llll}
\hline & $450 \mathrm{ppmv}$ & $550 \mathrm{ppmv}$ & $650 \mathrm{ppmv}$ \\
\hline Quotas $(\mathrm{GtC})$ & 426 & 542 & 564 \\
Probability of staying below $2^{\circ} \mathrm{C}$ & $50 \%$ & $20 \%$ & $5 \%$ \\
\hline
\end{tabular}

Notes: Own estimates based on:

${ }^{a}$ Simon Mueller, Climate and Environmental Physics, Physics Institute, University of Bern, see http://www.climate.unibe.ch/emicAR4/

${ }^{b}$ R. Knutti, F. Joos, A.S. Mueller, G.-K. Plattner, T.F. Stocker, Probabilistic climate change projections for stabilization profiles, Geophysical Research Letters, 32, 20, 21 October 2005

${ }^{\mathrm{c}} \mathrm{M}$. Meinshausen, On the risk of overshooting $2^{\circ} \mathrm{C}$, 2632nd Council meeting, Environment, Luxembourg, see http://www.stabilisation2005.com/

to uncertainties in climate sensitivity and ocean heat uptake. Previously published probability density functions of climate sensitivity are used to calculate probabilistic projections for different $\mathrm{CO}_{2}$ stabilization levels and to calculate the probability of not exceeding a certain global mean surface temperature for a given stabilization level. The resulting probabilities of staying below a certain equilibrium global mean surface warming for a given stabilization value of atmospheric $\mathrm{CO}_{2}$ equivalent radiative forcing are reproduced in Fig. $10 .^{5}$

The scenarios assumptions are summarized in Table 1.

5 All published climate sensitivity PDFs are explored for this graph. The authors propose another graph based on the more optimistic case where the 1.5 to $4.5 \mathrm{~K}$ sensitivity range is assumed to be the $90 \%$ confidence range of a log-normal distribution. 


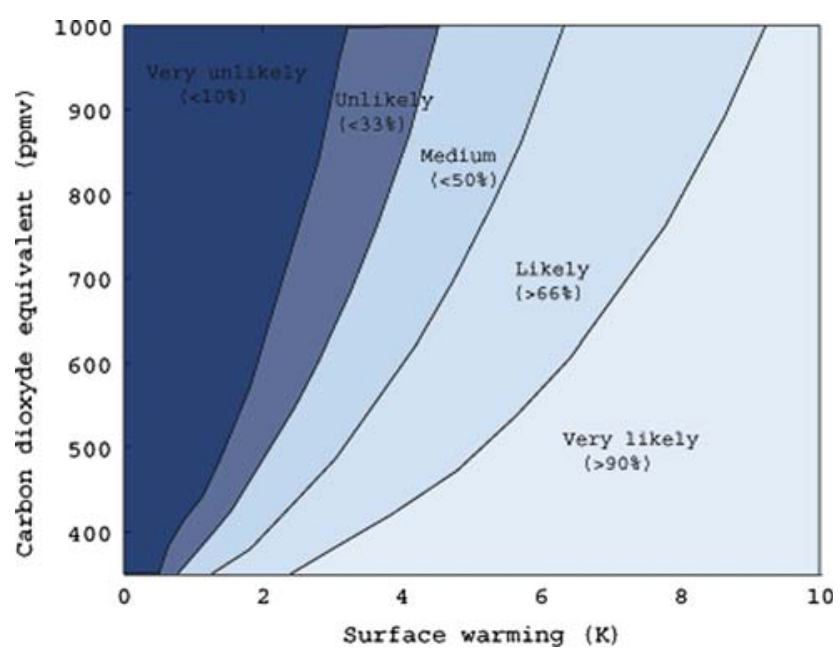

Fig. 10 Probability of staying below a certain equilibrium global mean surface warming for a given stabilization value of atmospheric $\mathrm{CO}_{2}$ equivalent radiative forcing for all published climate sensitivity PDFs

\subsection{Simulation results}

In Fig. 11, we present the worldwide GHG emissions pathways that correspond to non-cooperative equilibrium solutions under a global cumulative emissions constraint by 2050. We compare the coupled equilibrium in the deterministic and stochastic game framework. When one has to reach a 550 or $650 \mathrm{ppmv}$ concentration target by 2100 with certainty, GHG emissions increase linearly from $9 \mathrm{GtC}$ in 2000 to 12.2 and $12.9 \mathrm{GtC}$ in 2050 , respectively. In contrast, early action is required when the players are committed to stabilize at 450 ppmv. In that case, global GHG emissions are reduced at $7.8 \mathrm{GtC}$ in 2025 , and $8.8 \mathrm{GtC}$ in 2050 .

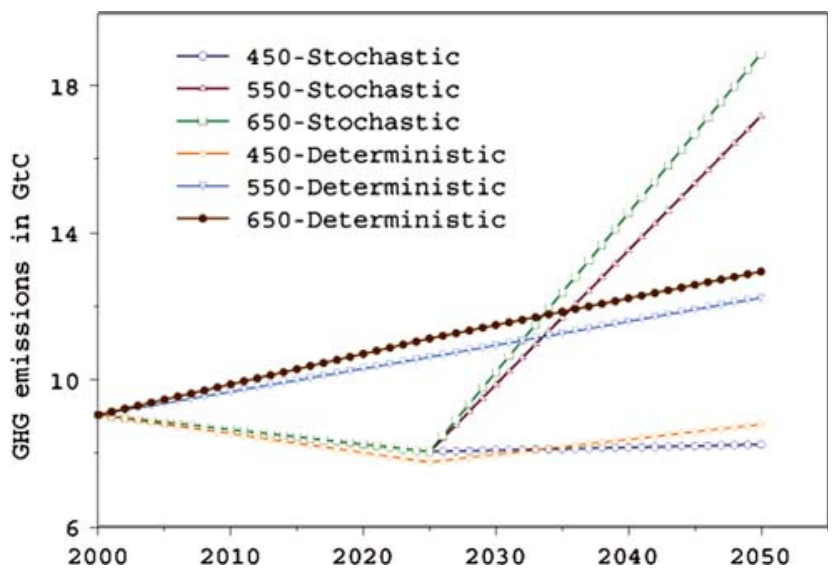

Fig. 11 Global emission path under uncertainty 


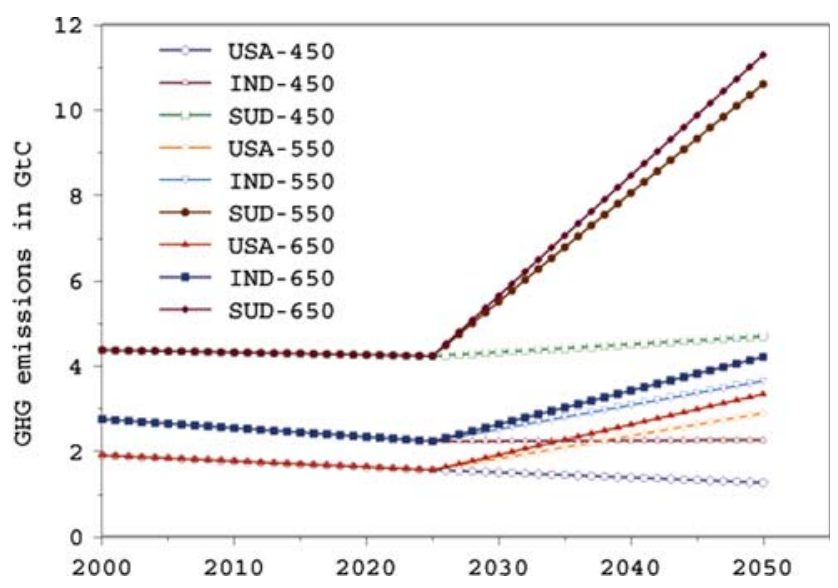

Fig. 12 Reduction efforts under uncertainty

Table 2 Equilibrium GHG emissions abatement by regions in 2025 (\% change from the BaU)

\begin{tabular}{llllll}
\hline & All GHGs & $\mathrm{CO} 2$ & $\mathrm{CH} 4$ & $\mathrm{~N}_{2} \mathrm{O}$ & F-gases \\
\hline USA & $-38 \%$ & $-37 \%$ & $-55 \%$ & $-18 \%$ & $-61 \%$ \\
IND & $-28 \%$ & $-26 \%$ & $-44 \%$ & $-17 \%$ & $-55 \%$ \\
SUD & $-44 \%$ & $-50 \%$ & $-34 \%$ & $-13 \%$ & $-64 \%$ \\
WORLD & $-39 \%$ & $-41 \%$ & $-38 \%$ & $-15 \%$ & $-61 \%$ \\
\hline
\end{tabular}

Table 3 Welfare change at the equilibrium by regions in 2025 (\% change from the $\mathrm{BaU}$ )

\begin{tabular}{lcccc}
\hline & Surplus & Terms of trade & Permits transfers & Deadweight loss \\
\hline USA & $-0.36 \%$ & $0.26 \%$ & $0.01 \%$ & $-0.63 \%$ \\
IND & $-0.56 \%$ & $0.13 \%$ & $-0.22 \%$ & $-0.48 \%$ \\
SUD & $-2.35 \%$ & $-0.84 \%$ & $0.43 \%$ & $-1.94 \%$ \\
\hline
\end{tabular}

In the stochastic game, as shown in Fig. 11, early abatement is needed in the first period of commitment with respect to the deterministic scenarios 550 and 650. In contrary when the real severity of climate change is known emission path can overtake those of the two deterministic scenarios in the second period of the simulation.

Figure 12 plots GHG emissions pathways by regions. As shown in the graph, GHG emissions are reduced by around $18 \%$ in USA and in other OECD countries at the equilibrium in the 2000-2025 period. Under the uncertainty about the stabilization target, developing countrie's GHG emissions would go from $4.38 \mathrm{GtC}$ in 2000 to $4.24 \mathrm{GtC}$ in 2025 representing a $3 \%$ reduction rate in the whole period of time.

In Table 2 we present GHG emissions abatement in the stochastic scenario for the different greenhouse gases described by the GEMINI-E3 model, $\mathrm{CO}_{2}$, methane and fluorinated gases would have significant reduction. Table 3 shows the welfare change and the components of this cost under the stochastic framework in 2025 for the three players. 


\section{Conclusion}

In this paper we have shown how to formulate and solve numerically a noncooperative game for the description of climate negotiations under a coupled constraint on long term carbon concentrations. We have also illustrated the use of an oracle based method to solve a variational inequality problem where the gradient operator is revealed by simulations performed with detailed economic models. The model can take into consideration the main sources of uncertainty concerning the climate sensitivity. The use of an equilibrium solution instead of a social optimum leads to solution which are self-enforcing in the sense that a group of country which agree on the necessity to limit the temperature change and which accepts the weighting of actors describing the distribution of burden will have no reason to deviate from the negotiated equilibrium solution.

Our policy scenarios are built on the objective of keeping global mean surface temperature relative to preindustrial levels below $2^{\circ}$. This target is in line with the European proposal. Our first simulations take into account three regions (USA, other OECD countries and developing countries) and two periods of commitment (2000-2025, 2025-2050). We show that with this concept of normalized equilibrium we could find solution where the welfare gain for developing countries is positive and so could motivate the participation of such group of countries to the second round of negotiation of the Kyoto protocol. One extension of the model would be to consider several groups of developing countries (China, India, Brazil, other DCs) and more periods in order to design more concrete schemes of climate change agreement.

In this first implementation we have restricted the model to a set of two periods and we have used a relatively simple description of the technological choices offered to developed and developing countries. In the forthcoming use of the method we plan to use technology-rich models like MARKAL-World. ${ }^{6}$ or TIMES, ${ }^{7}$ in conjunction with GEMINI-E3 to provide the evaluation of the players' payoffs in the oracle-based method.

\section{References}

Armington PS (1969) A theory of demand for products distinguished by place of production. IMF Staff Papers 16:159-178

Babonneau F, Beltran C, Haurie A, Tadonki C, Vial J-P (2006) Proximal-accpm: a versatile oracle based optimization method. In: Kontoghiorghes EJ (ed) Optimisation, econometric and financial analysis. Advances in computational management science, vol 9. Springer, Boston, pp 3-23

Bernard A, Haurie A, Vielle M, Viguier L (2005) A two-level dynamic game of carbon emissions trading between Russia, China, and Annex B countries. J Econ Dyn Control (submitted for publication)

\footnotetext{
6 See Labriet and Loulou (this issue).

7 See Loulou (this issue).
} 
Bernard A, Paltsev S, Reilly JM, Vielle M, Viguier L (2003) Russia's role in the Kyoto protocol. Report 98, MIT Joint Program on the Science and Policy of Global Change, Cambridge, MA, June

Bernard A, Vielle M (1998) La structure du modèle GEMINI-E3. Econ Prévis 5(136)

Bernard A, Vielle M (2000) Comment allouer un coût global d'environnement entre pays: permis négociables versus taxes ou permis négociables et taxes? Econ Int 2(82)

Bernard A, Vielle M (2003) Measuring the welfare cost of climate change policies: a comparative assessment based on the computable general equilibrium model GEMINI-E3. Environ Model Assessm 8(3):199-217

Bernard A, Vielle M, Viguier L (2005) Carbon tax and international emissions trading: a swiss perspective. In: Haurie A, Viguier L (eds) Coupling climate and economic dynamics. Springer, Heidelberg

Buchner B, Carraro C, Cersonimo I, Marchiori C (2005) Back to Kyoto? US participation and the linkage between $R \& D$ and climate cooperation. In: Haurie A, Viguier L (eds) The coupling of climate and economic dynamics. Springer, Dordrecht, pp 173-204

Carraro C, Siniscalco D (1996) R\&D cooperation and the stability of international environmental agreements. In: Carraro C (ed) International environmental negotiations. Kluwer Academic Publishers

Ferris MC, Munson TS (2000) Complementarity problems in GAMS and the PATH solver. J Econ Dyn Control 24:165-188

Ferris MC, Pang JS (1997) Complementarity and variational problems: state of the art. SIAM Publications, Philadelphia

Goffin JL, Haurie A, Vial J-Ph (1992) Decomposition and nondifferentiable optimization with the projective algorithm. Manage Sci 37:284-302

Haurie A (1995) Environmental coordination in dynamic oligopolistic markets. Group Decis Negotiat 4:46-67

Haurie A, Krawczyk J (1997) Optimal charges on river effluent from lumped and distributed sources. Environ Model Assessm 2:177-199

Haurie A, Moresino F (2002) S-adapted oligopoly equilibria and approximations in stochastic variational inequalities. Ann Oper Res 114:183-201

Haurie A, Moresino F, Viguier L (2005) A two-level differential game of international emissions trading. In: HAurie A, Raghavan TES (eds) Advances in dynamic games and applications, vol 8. Birkhäuser, Boston (in press)

Haurie A, Smeers Y, Zaccour G (1990) Stochastic equilibrium programming for dynamic oligopolistic markets. J Optim Theory Appl 66:243-253

Haurie A, Viguier L (2003) A stochastic game of carbon emissions trading. Environ Model Assessm 8(3):239-248

Haurie A, Zaccour G (1995) Differential game models of global environmental management. In: Carraro C, Filar JA (eds) Game-theoretic models of the environment. Annals of the International Society of Dynamic Games, vol 3. Birkhäuser, Boston, pp 3-23

Hertel TW (1997) Global trade analysis: modeling and applications. Cambridge University Press, Cambridge

IEA (2002) Beyond Kyoto. Energy dynamics and climate stabilisation. OECD/IEA, Paris

Luo Z-Q, Goffin J-L, Ye Y (1996) Complexity analysis of an interior cutting plane for convex feasibility problems. SIAM J Optim 6:284-302

Knutti R, Meehl GA, Allen MR, Stainforth DA (2006) Constraining climate sensitivity from the seasonal cycle in surface temperature. J Climate 19(17):4224-4233

Nesterov Y, Nemirovskii A (1994) Interior point polynomial algorithm in convex programming. SIAM, Philadelphia

Nesterov Y, Vial J-P (1999) Homogeneous analytic center cutting plane methods for convex problems and variational inequalities. SIAM J Optim 9(2):707-728

Philibert C (2000) How could emissions trading benefit developing countries? Energy Policy 28:947-956

Philibert C, Pershing J (2001) Considering the options: climate targets for all countries. Climate Policy 1:211-227

Rosen JB (1965) Existence and uniqueness of equilibrium points for concave $n$-person games. Econometrica 33(3):520-534 
Stone JRN (1983) Linear expenditure systems and demand analysis: an application to the pattern of British demand. Econ J 64:511-527

Toth FL (2005) Coupling climate and economic dynamics: recent achievements and unresolved problems. In: Haurie A, Viguier L (eds) The coupling of climate and economic dynamics. Springer, Dordrecht, pp 35-68 